Pteridines

Vol. 8, 1997, pp. $21-26$

\title{
Inhibitory Effects of Pteridines on the Cytochrome P-450 Enzyme System
}

\author{
Renate Horejsi, Jürgen Daczki, Reinhard Möller, Karl Öttl, Karoline Vrecko and Gilbert Reibnegger ${ }^{\S}$ \\ Institute for Medical Chemistry and Pregl Laboratory, Karl-Franzens-University Graz, Harrachgasse \\ $21 / \mathrm{II}$, A-8010 Graz, Austria
}

(Received February 10, 1997)

\begin{abstract}
Summary
The effects of 17 different pteridine derivatives on rat liver microsomal cytochrome P- 450 catalyzed degradation of aminoantipyrin were studied. Neopterin (41\% of control), biopterin (42\%), monapterin $(73 \%)$, pterin $(74 \%)$, lumazine $(75 \%)$ and 6-methylpterin $(77 \%)$ significantly inhibit the degradation of aminoantipyrin. 6,7-Dimethylpterin $(80 \%)$ and 6-hydroxymethylpterin $(89 \%)$ show weak but non-significant effects, and the remaining pteridine derivatives investigated (pterin-6-carboxylate, pterin-7-carboxylate, pterin-6-aldehyde, 7,8-dihydro-neopterin, 7,8-dihydro-biopterin, sepiapterin, xanthopterin, isoxanthopterin, and leukopterin) do not interact with the enzyme system. In addition, spectroscopic investigations were performed for colourless pteridine derivatives. Neopterin, biopterin, monapterin and pterin yielded type II binding spectra, indicating their binding to the heme iron of the active center of the enzyme. In agreement with the inhibition studies, 7,8-dihydroneopterin and 7,8-dihydrobiopterin did not exhibit spectroscopic evidence for their interaction with the enzyme system. When attempting to correlate structural features of the pteridine compounds with their effect on the cytochrome P450 system, presence of a hydroxy function at $C_{2}{ }^{\prime}$ of an alkyl sidechain at $C_{6}$ of the ring system was found to be particularly important for inhibition of aminoantipyrin degradation, while a 7,8-dihydro structure has the opposite effect. Interestingly, pteridines strongly interacting with the cytochrome P450 system also cause strong enhancement of radical-induced luminol-dependent chemiluminescence and of the growth-inhibitory potential of radical-vielding disinfectants towards bacteria, while 7,8-dihydropteridines in these other experimental systems show strong scavenger activity.
\end{abstract}

Key words : Cytochrome P-450, Rat liver microsomes, Pteridine derivatives, Molecular structure, Aminoantipyrin degradation

\section{Introduction}

The cytochrome P-450 dependent monooxygenases are a superfamily of heme proteins that catalyze the oxidative metabolism of a wide variety of endogenous and exogenous compounds (1-3). Cy-

§ Correspondence and requests for reprints should be addressed to: Prof. Gilbert Reibnegger tochrome P-450 enzymes are found in living cells of practically all species (4).

Unconjugated pteridines occur ubiquitously in living cells, but only little is known about their biochemical functions.Tetrahydrobiopterin-dependent hydroxylases are known since 1963 (5), and tetrahydrobiopterin was identified as essential requirement for proper functioning of the inducible nitric oxidase system (6), which is a cytochrome $\mathrm{P}$ - 
450 type hemoprotein (7). Neopterin is produced by human and primate macrophages after stimulation by interferon- $\gamma$. It is a useful indicator of cell mediated immune reactions in many diseases $(8)$.

A possibly important aspect of pteridine derivatives was only recently dicovered: Pteridines modulate free radical-induced growth inhibition in Escherichia coli bacteria. Aromatic pterins with polyhydroxylated alkyl side chains in position 6 of the pteridine ring system such as, e.g., neopterin, biopterin and monapterin enhance this growth inhibition, while their reduced counterparts with a 7 , 8-dihydrostructure (7,8-dihydrobiopterin, 7,8-dihydroneopterin, sepiapterin and others) strongly suppress growth inhibition (9).

A quite different experimental design employing chemiluminescence experiments resulted in a similar ranking of the same pteridine derivatives: aromatic pteridines enhance radical-induced light output in luminol-dependent chemiluminescence experiments, whereas reduced pteridine derivatives exert an opposite effect; they strongly suppress light output (10)

Stimulated by these and similar earlier observations pointing to a general role of pteridines in experimental systems involving free radicals or similarly activated species, and due to their chemical structures suggesting a possible role of pteridines as substrates or inhibitors of the cytochrome P450 enzyme system, the effects of 17 structurally different pteridines on rat liver microsomal P-450 preparations were investigated.

\section{Material and Methods}

\section{Animals}

Male Wistar rats (Zentrale Versuchstieranstalt Himberg, Austria) were housed in group cages under controlled room conditions constant temperature of $22^{\circ} \mathrm{C}$ and $70 \%$ humidity: a 12 hour's light-dark cycle). Rat laboratory chow Tagger Futtermittelwerke, Graz, Austria) and tap water were allowed ad libitum.

\section{Chemicals}

All pteridines were purchased from Schircks Laboratories (Jona, Switzerland) in the highest available purity degree. The chemical structures of the investigated pteridines are shown in Figure 1. Biochemicals were obtained from Sigma Chemical Co (St Louis, MO), other chemicals from E. Merck (Vienna, Austria).
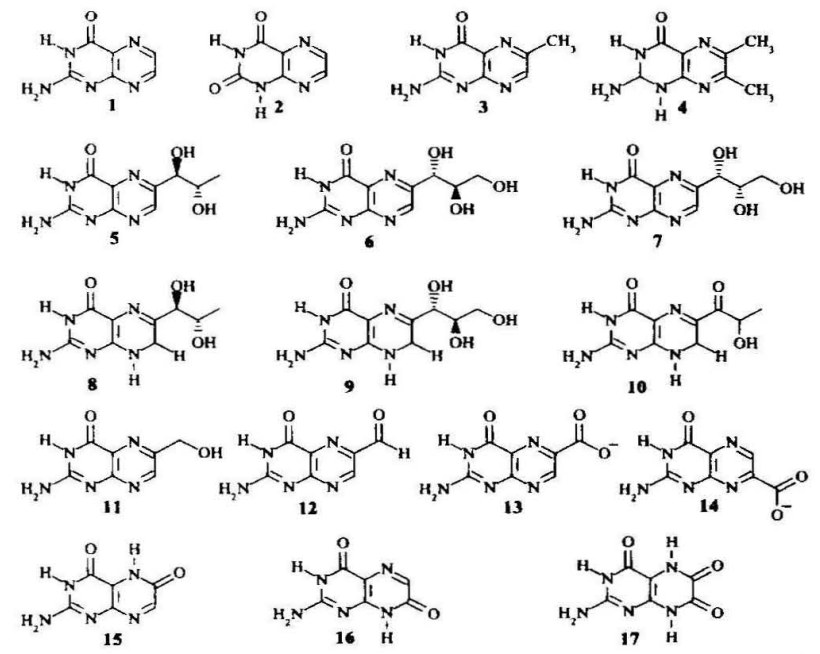

Figure 1. Chemical structures of the pteridines investigated. 1: pterin; 2: lumazine; 3: 6-methylpterin; 4: 6,7-dimethypterin; 5: biopterin; 6: neopterin; 7: monapterin, 8: 7,8dihydrobiopterin; 9: 7,8-dihydroneopterin; 10: sepiapterin; 11: 6-hydroxy-methylpterin; 12: 6-formylpterin; 13: pterin6-carboxylate; 14: pterin-7-carboxylate; 15: xanthopterin; 16: isoxanthopterin; 17: leukopterin.

\section{Preparation of microsomes}

Rat livers were homogenized in $0.025 \mathrm{M}$ Tris$\mathrm{HCl}$ buffer, $\mathrm{pH} 7.4$, containing $1.15 \%$ potassium chloride. Microsomes were prepared by differential centrifugation $(9000 \times \mathrm{g}$ for 20 minutes, 105 , $000 \times \mathrm{g}$ for 60 minutes and $105,000 \times \mathrm{g}$ for 90 minutes). Protein content of the microsomal suspension was measured by the Lowry procedure (11). The cytochrome P-450 concentration was estimated by differential spectroscopy (12) using a DL 640 spectrophotometer (Beckman, Palo Alto, (a).

\section{Binding spectra}

Two $\mathrm{ml}$ of the microsomal suspensions (about $\mathrm{l}$ mg protein per $\mathrm{ml}$ ) were mixed with $60 \mathrm{ml}$ of the pteridine solution ( $1 \mathrm{mg}$ per $\mathrm{ml}$ dimethylsulfoxide) at $22^{\circ} \mathrm{C}$ in a curette; binding spectra were recorded spectroscopically. Raw data were corrected by substracting the light absorbance of the separate substrates

\section{Enzyme assay}

Rat liver microsomes were incubated together with increasing concentrations $(40-320 \mathrm{nmol})$ of pteridine solution for 20 minutes. The assay contained about $5 \mathrm{mg}$ microsomal protein, $5 \mathrm{mmol}$ magnesium chloride, $8 \mathrm{mmol}$ isocitrate, $1 \mathrm{mmol}$ 
NADP and $8 \mathrm{mmol}$ aminoantipyrin. The yield of formaldehyde was estimated using the Nash-reagent (13).

\section{Statistical analysis}

Enzyme activities in the aminoantipyrin assay are expressed as nmol formaldehyde liberated per minute and $\mathrm{mg}$ protein. Values are mean values + SEM. for four (in the case of pteridines showing absolutely no inhibitory effect) or six assays from liver microsomes of different rats. Statistical significance of the effects of the pteridines were tested by analysis of variance. As dependent variable we chose measured mean values on formaldehyde liberation after $20 \mathrm{~min}$ incubation time from four to six separate assays, and the concentration of the pteridine derivative under investigation was chosen as grouping variable.

In order to obtain an estimate which structural features of the pteridine molecules investigated were responsible for the observed effects on the aminoantipyrin assay, multiple linear regression analysis was used aimed at construction of a simple multivariate index permitting prediction of the effect of a given pteridine compound on the basis of its particular chemical structure. This was achieved by the following procedure: each interesting structural feature was coded into a binary variable indicating either absence, or presence of this feature. Table 1 explicates this binary coding of chemical structure. Thus, each pteridine can be represented by a string of 12 binary variables. The importance of each binary variable taken separately was assessed by the Mann-Whitney $\mathrm{U}$ test. These binary variables were then used as independent variables in a multiple linear regression analysis; the observed inhibitory effect of the compounds on aminoantipyrin degradation was chosen as dependent variable. By "all possible subsets regression" the combination of structural features permitting optimum prediction of the observed effects was identified. Selection of the "best subset" was performed by searching for the subset of binary variables yielding the smallest value of the Mallow's Cp-statistic (14) by which the subset is selected with the maximum squared multiple correlation while employing at the same time a penalty function discouraging the inclusion of too many variables in the model. Thus, the technique attempts to identify subsets that permit good prediction but are also parsimonious. In view of the relatively limited number of compound this seemsclearly desirable. The same procedure was used in earlier publications on effects of various pteridines

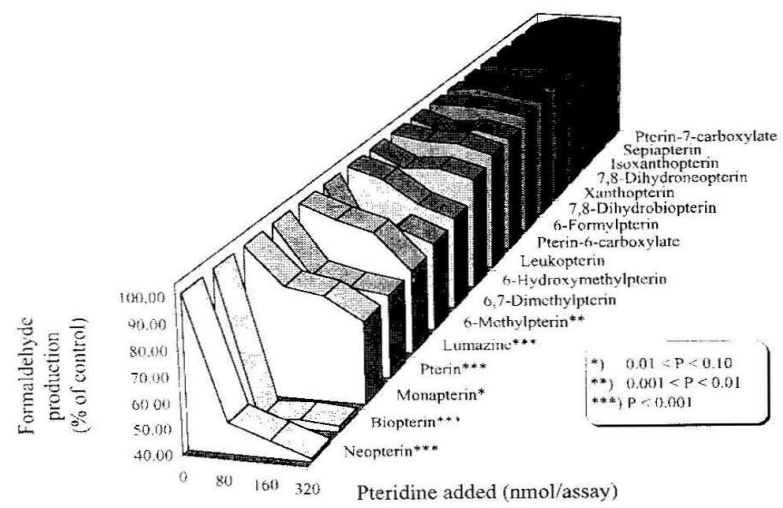

Figure 2. Effects of pteridines on cytochrome P450-mediated degradation of aminoantipyrin. Shown are mean effcets of four to six separate experiments using different concentrations of pteridines for an incubation time of $20 \mathrm{~min}$ utes.

on cytotoxicity of chloramine $\mathrm{T}$ on $E$. coli bacteria (9) and on experiments probing the chloramine $\mathrm{T}$-induced luminol-dependent chemiluminscence (10).

\section{Results}

The mean amount of cytochrome P450-enzyme protein per assay was $1.82(\mathrm{SEM}=0.094) \mathrm{nmol}$ per mg microsomal protein.

Effects of pteridine derivatives on the degradation of aminoantipyrin by cytochrome P450

Figure 2 summarizes the results of the inhibition studies of cytochrome P450-mediated degradation of aminoantipyrin in the presence of the various pteridine derivatives. As the figure demonstrates there were two groups of pteridines with respect to their effect of the enzyme-catalyzed reaction: the aromatic compounds neopterin, biopterin, monapterin, pterin, lumazine and 6-methylpterin exerted statistically significant inhibition of aminoantipyrine degradation, while effects of the remaining substances were weak or completely negligible, and in neither case reached statistical significance.

Correlation between structural features of pteridines and their effects on cytochrome P450-mediated degradation of aminoantipyrin

Table 1 explains the coding of important structural features into binary variables and also shows how each of these characteristics, taken separately, influences the observed inhibition of aminoanti- 
Table 1. Coding of structural features of the pteridines studied, and their association with the inhibition of cytochrome P450-mediated degradation of aminoantipyrin

\begin{tabular}{|c|c|c|}
\hline Variable & Possible Value & P-value* \\
\hline Structural class & $0=$ pterin,$\quad 1=$ lumazine & 0.41 \\
\hline 7,8-dihydro structure & $0=$ no, $1=$ yes & 0.059 \\
\hline alkyl group at $C_{t}$ & $0=$ no, $\quad l=$ yes & 0.33 \\
\hline alkvl group at $C_{7}$ & $0=\mathrm{no}, \mathrm{l}=\mathrm{yes}$ & 0.68 \\
\hline oxo group at $C_{6}$ & $0=$ no, $1=$ yes & 0.58 \\
\hline oxo group at $C_{7}$ & $0=$ no, $1=$ yes & 0.22 \\
\hline caboxylate group at $C_{6}$ & $0=$ no, $1=$ yes & 0.84 \\
\hline carboxylate group at $\mathrm{C}_{7}$ & $0=$ no, $1=$ yes & 0.10 \\
\hline hydroxy group at $C_{1}^{\prime}$ & $0=$ no, $1=$ yes & 0.16 \\
\hline hydroxy group at $C_{2}$ & $0=$ no, $1=$ yes & 0.55 \\
\hline hydroxy group at $\mathrm{C}_{3}{ }^{\prime}$ & $0=$ no, $1=$ yes & 0.26 \\
\hline oxo group at $C_{1}$ & $0=$ no, $\quad 1=$ yes & 0.18 \\
\hline
\end{tabular}

* Significance of effects (Mann-Whitney U test)

pyrin degradation by the microsomal preparations. On the basis of the $\mathrm{P}$ values reported in Table 1 , only one single structural feature was found exerting a significant effect; namely, whether a given pteridine is aromatic or reduced.

In contrast, in the multiple linear regression analysis a combination of two structural features was found to permit optimum prediction of the inhibitory effect of a pteridine derivative, namely, presence versus absence of the 7,8-dihydro structure (F-to-remove statistic 25.9; $\mathrm{P}<0.0001$ ) and presence versus absence of a hydroxy group at the $\mathrm{C}_{2}{ }^{\prime}$ atom of an alkyl side chain situated at the $\mathrm{C}_{6}$ atom of the pteridine ring system (F-to-remove statistic $24.8 ; \mathrm{P}<0.0001)$. The regression coefficient of the binary variable coding for the distinction aromatic versus reduced compound was 46.8 ( $\mathrm{SE}, 9.2$ ); the regression coefficient for the binary variable coding for the presence versus absence of the hydroxy group was -36.5 (SE, 7.3).

To predict the effect on the aminoantipyrin degradation experiment of a given pteridine derivative, we may use this simple model in the following way: if neither of the two important structural features is present, our estimate is 100 percent, indicating that there is no effect. Presence of the hydroxy group at the specified atom (for example, neopterin, biopterin, monapterin) leads to the prediction $100-36.5=63.5$ percent; i.e., a marked inhibitory effect. Simultaneous presence of an hydroxy function at $\mathrm{C}_{2}{ }^{\prime}$ and a 7,8-dihydro structure, however, leads to the prediction of practically no net effect, since $100-36.5+46.8=110.3$ percent approximates 100 percent, and in the framework of this simple model, means no effect. This situation is found, e.g, with 7,8-dihydroneopterin, 7,8dihydrobiopterin and sepiapterin.
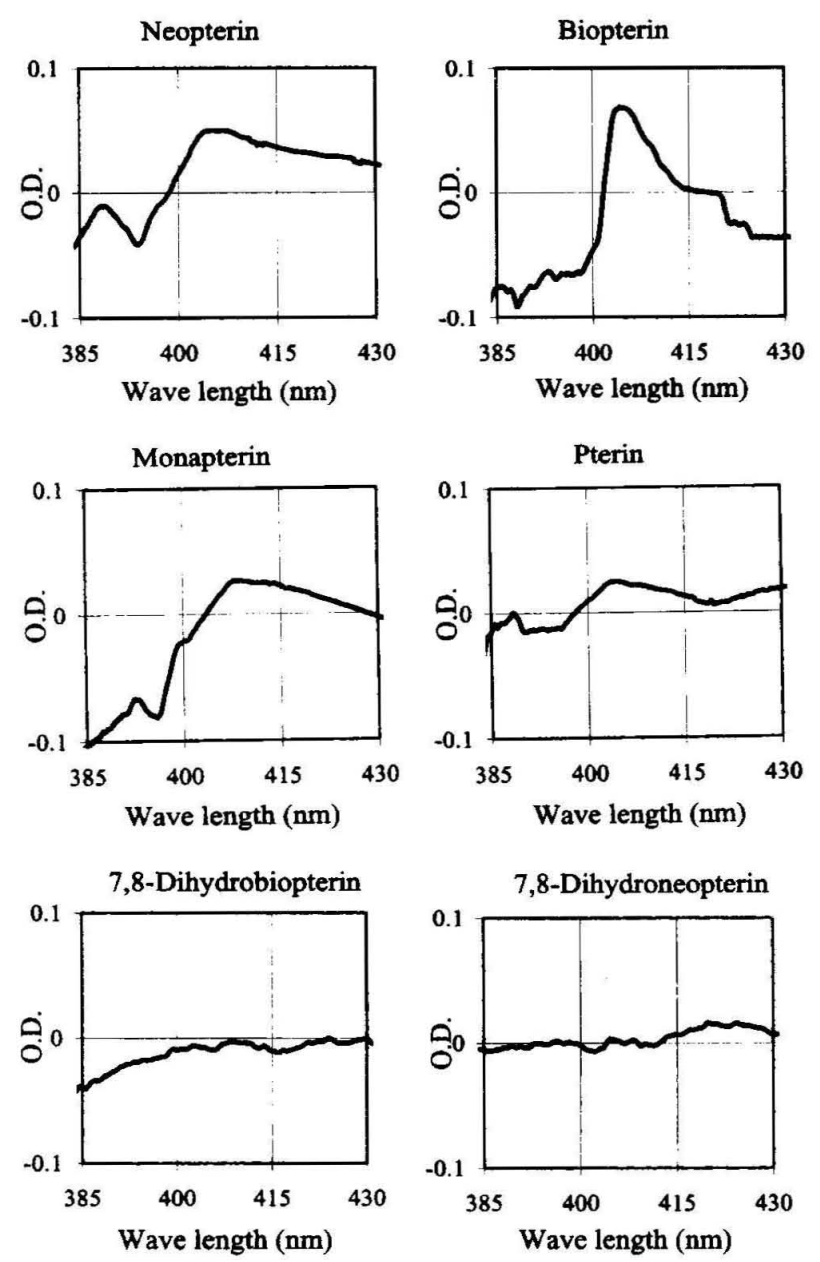

Figure 3. Difference spectra demonstrating the interaction of the active site of the cytochrome P450 system and pteridines.

\section{Type II binding spectra}

Because of the yellowish colour with absorption maxima between 380 and $300 \mathrm{~nm}$ recording of a distinguished binding spectrum of several pteridines with microsomal preparations was impossible. Figure 3 shows the spectra obtained with four aromatic and two reduced pteridine derivatives, namely, neopterin, biopterin, monapterin and unsubstituted pterin, and 7,8-dihydroneopterin as well as 7,8-dihydrobiopterin.

The observed type II spectra indicate for neopterin, biopterin and monapterin a strong interaction with the cytochrome $\mathrm{P} 450$ system; for pterin a similar but weaker interaction is suggested. In marked contrast, both reduced pteridine compounds do not seem to interact with the enzyme system at all; no evidence for binding of the compounds to the active site of the cytochrome $\mathrm{P}$ 450 system is observed. 


\section{Discussion}

Dependent on their chemical structures, pteridine derivatives show significant interactions with rat microsomal cytochrome $\mathrm{P} 450$ preparations. We have studied these interactions by two different designs; firstly, we have studied the effects of pteridines on the cytochrome P450-mediated degradation of aminoantipyrin; secondly, we have tried to investigate the interactions between certain pteridines and the enzyme system by spectroscopical technique.

The experiments exploring the effects of various pteridines upon the cytochrome P450-mediated aminoantipyrin degradation havé led to an interesting result: aromatic pteridines with a hydroxy group at the $\mathrm{C}_{2}{ }^{\prime}$ atom of the alkyl side chain located at $\mathrm{C}_{6}$ of the pteridine ring system show the strongest inhibitory effect. In contrast, other pteridine compounds show only weak or no effects, and a 7,8dihydro structure even annihilates the effect of the hydroxy group at $C_{2}$ '.

We do not vet fully understand the molecular interactions of the pteridines with the cytochrome $P$ 450 system but it is exiting to note that these very same molecular features were found to be the most important structural elements governing the interaction of different pteridines with wholly different systems; namely, their effects on chloramine-T mediated luminol-dependent chemiluminescence (10) and on chloramine-T mediated growth inhibition on E. coli bacteria (9). In these different experimental settings pteridines with the mentioned specific hydroxy function strongly enhance the effect of the free radical generator chloramine- $T$, while the 7,8 dihydro structure had the opposite effect.

Pteridines are frequently yellowishly coloured substances and show an absorption of light preferentially at wavelengths of 430 and less $\mathrm{nm}$. The spectra obtained with neopterin, biopterin, monapterin and-less pronouncedly-with pterin itself suggest that an interaction of the pteridines with the active center of the enzyme occurs. The measured difference spectra deviate slightly from an ideal expected curve, but they can be recognized unequivocally as type II spectra; the change of sign of the difference spectra at a wavelength of about 400 $\mathrm{nm}$ is distinctly seen at least for neopterin, biopterin and monapterin. Moreover, the spectra obtained are in accordance with the findings obtained with the aminoantipyrin system.

Current investigations probing the interactions of pteridines and various free radical by electron spin resonance techniques unequivocally demon- strate that reduced pteridines with a 7,8 -dihydro structure are indeed scavengers of free radicals, probably via their strongly reducing capacity. No explanation, however, has been obtained hitherto by these experiments for the enhancer effect of aromatic pteridines possessing a hydroxy group at $\mathrm{C}_{2}{ }^{\prime}$ of their side chain.

On the basis of recent studies on the interactions of pteridines with free radicals and activated species one is tempted to speculate for example that the teleological reason for the unique characteristic of human and primate macrophages to synthesize and release neopterin after stimulation by interferon- $\gamma$ could be an enhancer effect of this particular pteridine on the destructive free radicals produced by activated effector macrophages. This might seem even more reasonable in the light of the fact that in contrast to macrophages from other species such as mice or rats the human macrophage does not produce tetrahydrobiopterin in comparable quantities after stimulation, and it also does not respond to the stimulation with an equally strong production of nitric oxide as compared to the macrophages from other species.

To verify or falsify such speculations, further experiments using both electron spin resonance and mass spectroscopic techniques are scheduled with the aim to elucidate the chemistry of the interactions between pteridines and free radicals.

\section{References}

1. Kappus, H., Overview of enzyme systems involved in bioreduction of drugs and in redox-cycling, Biochem. Pharmacol 1986; 35: 1-6.

2. Hollebone, B.R., Categorization of lipophilic xenobiotics by the enthalphic structure-function response of hepatic mixed function oxidase, Drug Metab. Rev. 1986; 17: 93-143.

3. Newton, D. J., Wang, R. W., and Lu, A.Y. H., Cytochrome P-450 inhibitors: evaluation of specificities in the in vitro metabolism of therapeutic agents by human liver miscrosomes, Drug Metab. Disp. 1995; 154-158.

4. Nebert, D. W., and Gonzalez, F. J., P450 genes: structure, evolution, and regulation, Ann. Rev. Biochem. 1987; 56: 945-993.

5 . Kaufmann, S. The structure of phenylalanine hydroxylation cofactor, Proc. Natl. Acad. Sci. USA 1963; 50: 1085-1093.

6. Tayeh, M. A., and Marletta, M.A., Macrophage oxidation of $\mathrm{L}$-arginine to nitric oxide, nitrite and nitrate. Tetrahydrobiopterin is required as a cofactor, J. Biol. Chem. 1989; 264: 19654-19658.

7. White, K.A. and Marletta, M.A., Nitric oxide synthase is a cytochrome P-450 hemoprotein, Biochem- 
istry 1992; 31: 6627-6631.

8. Wachter, H., Fuchs, D., Hausen, A. Reibnegger, G., Weiss, G., Werner, E.R., and Werner-Felmaver G. Neopterin: Biochemistry-Methods-Clinical-Application. Berlin, NewYork: Walter de Gruyter, 1992.

9. Horejsi, R., Estelberger, W., Mlekusch, W., Möller, R., Öttl, K., Vrecko, K. and Reibnegger G. Effects of pteridines on chloramine-T-induced growth inhibition in E. Coli strains: correlations with molecular structure, Free Rad. Biol. Med. 1996; 21(2): 133-138.

10. Reibnegger, G., Fuchs, D., Murr, C., Dierich, M. P., Pfleiderer, W., and Wachter, H., Effects of pteridines on luminol-dependent chemiluminescence induced by chloramine-T Free Rad. Biol. Med. 1995; 18: 525523.

11. Lowry, O. H., Rosebrough, N. J., Farr, A. 1. and Randall, R. J., Protein measurement with the Folin phenol reagent., J. Biol. Chem. 1951; 193: 265-275.
12. OmuraT. and Sato, R., The carbon monoxide-binding pigment of liver microsomes, J. Biol. Chem. 1964; 239: 2370-2378.

13. Nash, T., The colorimetric estimation of formaldehyde by means of the Hantzsch reaction, Biochem. J. 1953; 55: 416-421

14. Daniel, C. and Wood FS. Fitting equations to data, Wiley, New York, 1971.

15. Kojima, S., Icho, T., Mori, H. and Arai, T., Enhancing potency of neopterin toward B-16 melanoma cell damage induced by UV-A irradation and its possible application for skin tumor treatment, Anticancer Res. 1995; 15: 1975-1980.

16. Hoffmann, G., Schobersberger, W., Frede, S., Pelzer, L., Fandrey, J., Wachter, H., Fuchs, D. and Grote, J., Neopterin activates transcription factor $-k \mathrm{~B}$ in vascular smooth muscle cells, FEBS Lett. 1996; 391 : 181184. 